\title{
Extracting Vibration Characteristics and Performing Sound Synthesis of Acoustic Guitar to Analyze Inharmonicity
}

\author{
Johnson Clinton' ${ }^{1}$ Kiran P. Wani ${ }^{2}$ \\ ${ }^{1} \mathrm{M}$ Tech (Auto. Eng.), VIT-ARAI Academy, Bangalore, India \\ ${ }^{2}$ ARAI Academy, Pune, India \\ Email: clinton.j2019@vitstudent.ac.in
}

How to cite this paper: Clinton, J. and Wani, K.P. (2020) Extracting Vibration Characteristics and Performing Sound Synthesis of Acoustic Guitar to Analyze Inharmonicity. Open Journal of Acoustics, 10, 41-50.

https://doi.org/10.4236/oja.2020.103003

Received: July 30, 2020

Accepted: September 27, 2020

Published: September 30, 2020

Copyright $\odot 2020$ by author(s) and Scientific Research Publishing Inc. This work is licensed under the Creative Commons Attribution International License (CC BY 4.0).

http://creativecommons.org/licenses/by/4.0/

\begin{abstract}
The produced sound quality of guitar primarily depends on vibrational characteristics of the resonance box. Also, the tonal quality is influenced by the correct combination of tempo along with pitch, harmony, and melody in order to find music pleasurable. In this study, the resonance frequencies of the modelled resonance box have been analysed. The free-free modal analysis was performed in $A B A Q U S$ to obtain the modes shapes of the un-constrained sound box. To find music pleasing to the ear, the right pitch must be set, which is achieved by tuning the guitar strings. In order to analyse the sound elements, the Fourier analysis method was chosen and implemented in $M A T L A B$. Identification of fundamentals and overtones of the individual string sounds were carried out prior and after tuning the string. The untuned strings showed irregular fundamental frequencies and higher partials of decay. Octaves and power spectrums have been presented and discussed in this paper.
\end{abstract}

\section{Keywords}

Modal Analysis, Resonance Frequency, Fast Fourier Transform, Octaves, Acoustic Guitar

\section{Introduction}

The musical instrument guitar is a system of complex vibrating elements. It is an assembly of several individual parts such as the body, sound hole, saddle, bridge, neck, headstock, tuning pegs and strings [1]. The sound energy is mainly produced by the sound box, also called as resonance box, which is propagated 
through the sound hole. The sound box itself is made of sub parts like sound board (or top-plate), side-board, tail bock, transversal and longitudinal bars and bottom panel [1] [2].

As the instrument is being played, the metal strings produce only a minor part of sound directly. Whereas, they cause the bridge and top plate (sound board) to attain an excited condition, transferring the acoustic energy to the air-cavity, bars, and bottom plate which contribute to the major part of the sound produced [3]. Hence, analysis of structural modes is vital in designing and optimizing the overall sound quality and performance of acoustic guitars.

Natural and resonant frequency along with the modal patterns of the guitar panels has been studied by researchers, but the approach followed varies based on analysis objective, followed methodology and material features. Previously, work has been done with the guitar box on electromechanical input-output parameters at multi-point locations on the top-plate and their correlation with acoustic efficiency, but the modal parameters are not analysed [4]. The use of force hammer as excitation source to generate vibration modes is a technique to analyse the structure [5] [6]. This method requires extensive experimental setup. The dynamic behaviour of individual plates in isolation was investigated using finite element method (FEM), but results indicated a deviation from the holistic analysis of the resonance box as a single unit [7] [8].

Sound wave analysis can bring about insights on elements of musical note. It has been found frequency or pitch of sound wave is dependent on the string tension, plucking style and location [9]. The classical approach to describe a musical note is the Fourier series [10]. By considering a sound signal $f(t)$, defined in the interval $(0, \Omega)$, its Fourier series is:

$$
c_{o}+\sum_{n=1}^{\infty}\left(a_{n} \cos \frac{2 \pi n t}{\Omega}+b_{n} \sin \frac{2 \pi n t}{\Omega}\right)
$$

where, coefficients $c_{o}, a_{n}, b_{n}$ are:

$$
\begin{gathered}
c_{o}=\frac{1}{\Omega} \int_{0}^{\Omega} f(t) \mathrm{d} t, \\
a_{n}=\frac{2}{\Omega} \int_{0}^{\Omega} f(t) \cos \frac{2 \pi n t}{\Omega} \mathrm{d} t, n=1,2,3, \cdots \\
b_{n}=\frac{2}{\Omega} \int_{0}^{\Omega} f(t) \sin \frac{2 \pi n t}{\Omega} \mathrm{d} t, n=1,2,3, \cdots
\end{gathered}
$$

Each term $\left\{a_{n} \cos (2 \pi n t / \Omega)+b_{n} \sin (2 \pi n t / \Omega)\right\}$ has a fundamental period of $\Omega / n$, and hence a frequency in time of $n / \Omega$. Advanced techniques include performing fast Fourier transform (FFT) analysis, a digital method to extract Fourier spectrums and analyse the signals in time domain [10] [11]. In the time domain, the signal or function's value is understood for all real numbers at various separate instances in the case of discrete-time or the case of continuous-time. It converts a time function into an integral of sine-waves of various frequencies or sum, each of which symbolizes a frequency component. The so-called spectrum of frequency components is the frequency-domain depiction of the signal. 
The current work involves the study of sound box, by initially making a computer aided design (CAD) and extracting the mode shapes using FEM to ascertain resonance characteristics. Further, spectrum analysis is carried out in $M A T L A B$ to output the time domain plots of sound emitted by tuned strings to that of untuned ones.

\section{Methodology}

\subsection{Design of Sound Box}

The CAD model of the guitar was designed in Solid Edge, a 3-D product design software. The dimensions of the guitar were as measured from Clayton DC41NT acoustic guitar (Figure 1). The top plate, side ribs, bottom plates and sound hole were modelled as per dimensions depicted in Figure 2. A flat-plate structure was chosen with $5 \mathrm{~mm}$ thickness. The individual components were assembled using face-to-face constrains to create a unibody structure similar to that of physical guitar construction (Figure 3). For the analysis work done in this paper, internal braces were neglected.

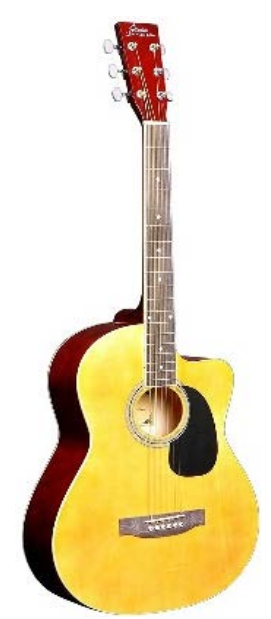

Figure 1. Clayton MC39CNT acoustic guitar.

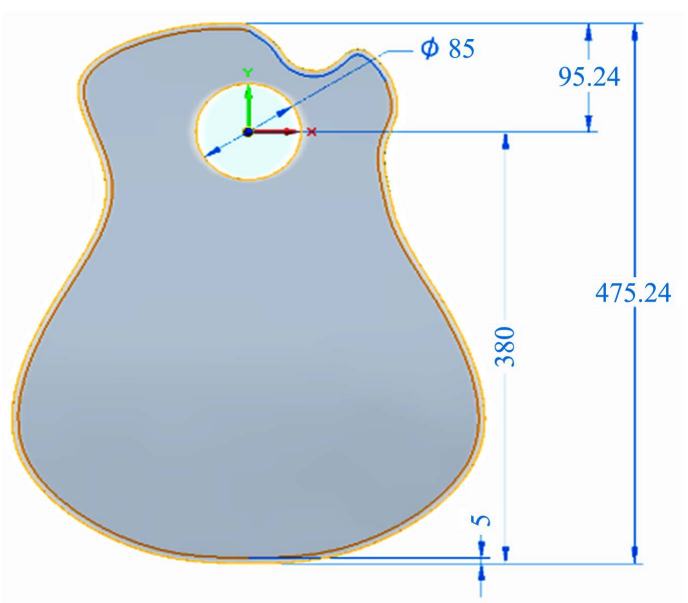

Figure 2. Model dimension (mm) 


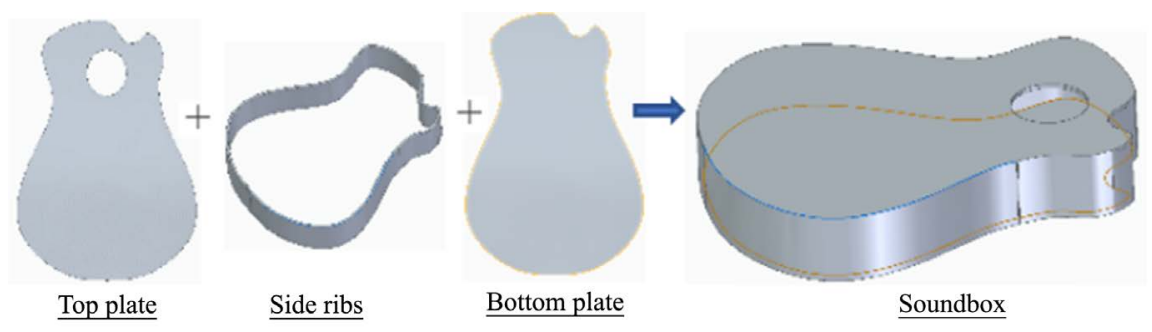

Figure 3. Assembled parts of soundbox.

\subsection{Modal Analysis Simulation}

The vibration modes of guitar primarily denote the actual deformation of the top-plate and back-plate of a guitar body [12]. Hence, only the sound box was considered for the modal study. The free-free modal analysis was carried out in $A B A Q U S$, a commercially available computer aided engineering (CAE) and finite element analysis (FEA) software. The CAD model of sound box was imported and material properties was assigned. Rosewood is an orthotropic material type in which the grain properties vary along the $\mathrm{x}, \mathrm{y}$ and $\mathrm{z}$ directions unlike isotropic materials as in Figure 4. Viscoelasticity is a unique property of wood, that the mechanical behaviour is time dependent where at any time instance, its performance will be a function of its past load history [13]. The materials properties of rosewood were selected from previous literature [13], and are listed in Table 1.

Boundary conditions where set as unconstrained to simulate the free-free modal analysis. Meshing was done using 2nd order 3-Dimensional stress Element type: Tet and Hex, consisting of 15834 elements (Figure 5). Linear Perturbation step was implemented in Abaqus and the frequency analysis was performed. The outputs of mode shapes and eigen frequencies were extracted.

\subsection{Sound Wave Analysis}

The Fourier transform can be utilized as a tool for conducting frequency and power spectrum analysis of time-domain signals. Fourier transform is a mathematical formula deriving the correlation between a signal sampled in time or space to the same signal sampled in frequency. In signal processing, the Fourier transform gives essential data about signal characteristics, importantly its frequency components. The Fourier transform is defined for a vector $x$ with $n$ uniformly sampled points as:

$$
y_{k+1}=\sum_{j=0}^{n-1} \omega^{j k} x_{j+1}
$$

where, $\omega=\mathrm{e}^{-2 \pi i / n}$ is one of $n$ complex roots of unity; $i$ is the imaginary unit; $x$ and $y$, the indices $j$ and $k$ range from 0 to $n-1$.

The $f f t$ function in MATLAB was used with a fast Fourier transform algorithm to compute the Fourier transform of guitar sound. Initially, the sound generated by the 6 strings $\mathrm{E}, \mathrm{A}, \mathrm{D}, \mathrm{G}, \mathrm{B}$, and $\mathrm{E}$ where recorded by a microphone in isolation, in an untuned state of the guitar. Then, the guitar strings were tuned with 


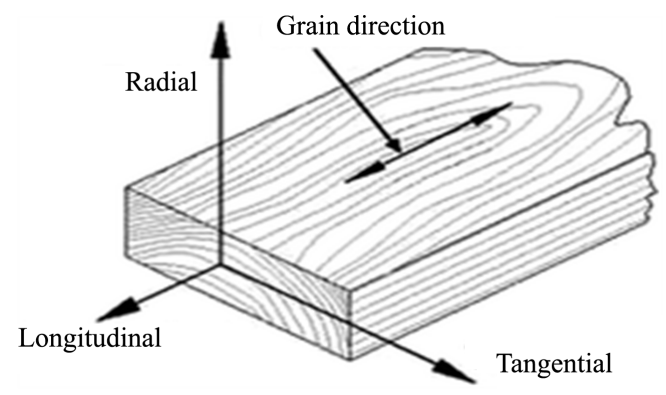

Figure 4. Property variation due to Grains in wood. Mirianon F, Fortino S. and Toratti T, 2008. A method to model wood by using ABAQUS finite element software. Helsinki: VTT Technical Research Centre of Finland.

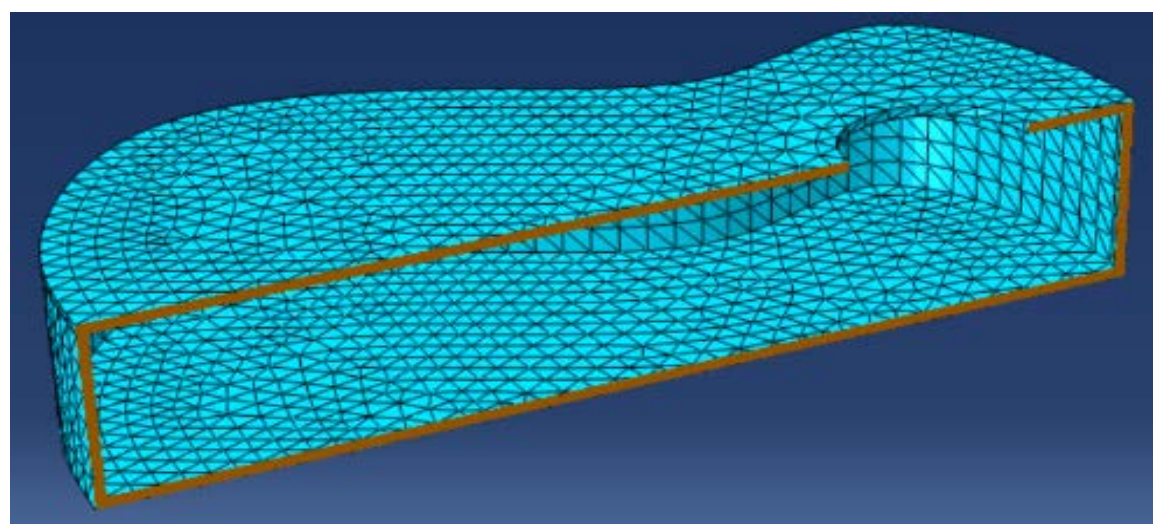

Figure 5. Cut section view of meshed sound box.

Table 1. Material properties of wood assigned in Abaqus. Mirianon F, Fortino S. and Toratti T, 2008. A method to model wood by using ABAQUS finite element software. Helsinki: VTT technical research centre of Finland.

\begin{tabular}{|c|c|}
\hline \multicolumn{2}{|c|}{ Material: Rosewood } \\
\hline Property & Value \\
\hline \multirow{3}{*}{ Youngs Modulus (MPa) } & $E_{1}=900$ \\
\hline & $\mathrm{E}_{2}=500$ \\
\hline & $\mathrm{E}_{3}=12000$ \\
\hline \multirow{3}{*}{ Poisson's Ratio } & $\mathrm{N}_{1}=0.297$ \\
\hline & $\mathrm{N}_{2}=0.641$ \\
\hline & $\mathrm{N}_{3}=0.604$ \\
\hline \multirow{3}{*}{ Shear Modulus (MPa) } & $\mathrm{G}_{1}=40$ \\
\hline & $\mathrm{G}_{2}=700$ \\
\hline & $\mathrm{G}_{3}=700$ \\
\hline Density $\left(\mathrm{Kg} / \mathrm{m}^{3}\right)$ & 450 \\
\hline
\end{tabular}

MUSEDO T-40 digital tuner, and the clear sound produced were recorded again. These audio signals recorded were extracted into $M A T L A B$ in . wav file format for analysis. 
The input audio file is resized to covert the sample size to a multiple of power of 2 . This can make the transform computation significantly faster, particularly for sample sizes with large prime factors. Then, $f f t$ function is used to compute the Fourier transform using the new signal length. The power spectrums of signal were plotted to compare the fundamental frequencies and harmonics variation caused by tuning the strings. Also, the octave spectrum of the signal was plotted using the poctave function by specifying bands per octave ( $b p o$ ).

\section{Results}

\subsection{Modal Analysis}

The modal shapes (also termed Chladni pattern) of the sound box plates are given by the distribution of the significant nodal lines on the surface of the structure. The nodal line represents the points or the areas which remain in an equilibrated position during vibration [14]. During vibrations, each pattern of the strutting system, characteristically, has nodes and antinodes at various locations on the body of the guitar [15]. An eigen solution performed on the model by simulation using the finite element solver yielded the natural frequency and mode shapes of the guitar shown in Figure 6 .

The acoustic performance of the classical guitar is given by the interaction between the top, back and side plates and the enclosed air. Due to the geometrical shape of the guitar and to the presence of the strutting system (in what number, pattern, material and dimensions are concerned), the inside volume of the air has an indefinable shape. Acoustically speaking, the irregular form presents numerous zones with a wide range of natural frequencies able to respond to forced vibrations as observed in the simulation.

The red regions depict strongest vibrations, yellow indicating lower vibration intensity, followed by green with weaker and blue depicting no vibrations. The first natural vibration mode was observed at $80 \mathrm{~Hz}$ and vibration modes with

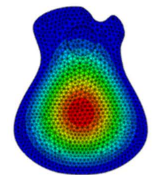

1: $80 \mathrm{~Hz}$

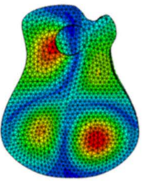

7: $217 \mathrm{~Hz}$

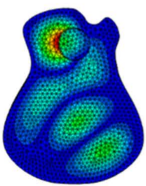

13: $322 \mathrm{~Hz}$

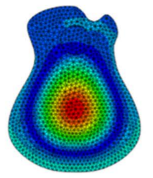

2: $105 \mathrm{~Hz}$
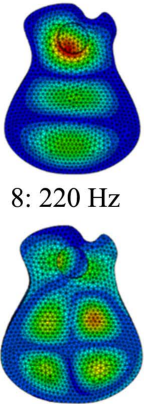

14: $335 \mathrm{~Hz}$
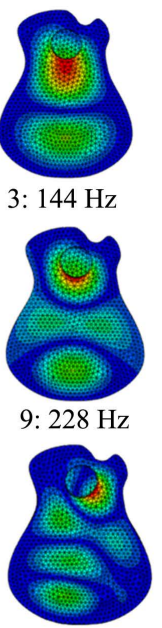

15: $345 \mathrm{~Hz}$
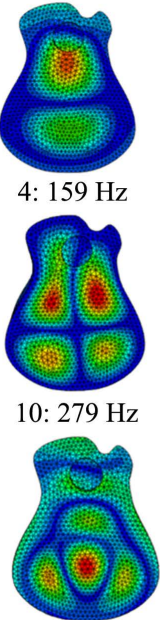

16: $385 \mathrm{~Hz}$
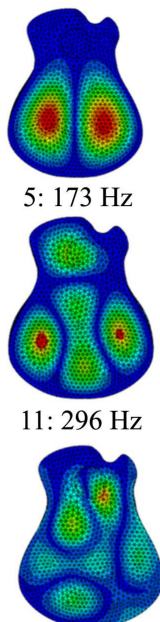

17: $398 \mathrm{~Hz}$

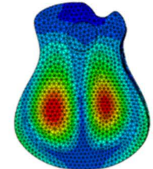

6: $188 \mathrm{~Hz}$

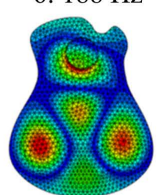

12: $303 \mathrm{~Hz}$

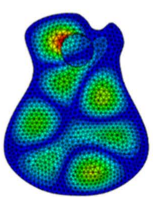

18: $414 \mathrm{~Hz}$

Figure 6. Mode shapes and natural frequency of guitar sound box. 
increasing of the frequency, become more complex and different from one structure to another.

\subsection{Sound Wave Analysis}

Inharmonicity of a nearly harmonic sound may be perceived in different ways, depending on the type and the amount of inharmonicity and the type of sound. In the guitar, however, the importance of inharmonicity to the quality of the sound has been unknown. According to current knowledge, small amounts of inharmonicity are perceived based on a timbral effect, not on segregating partials or a pitch change.

In order to modify the inharmonicity of a guitar sound, the frequencies of the partials have to be shifted without changing any other perceptual features of the signal. The fundamental frequencies of the tuned strings where obtained from FFT plot with parameters in Table 2. Frequency of first-string $E$ was lowest with $79 \mathrm{~Hz}$, followed by $A, D, G, B$ and $e$ as seen in Figure 7. Thick strings with large diameters vibrate slower and have lower frequencies than thin ones. String $e$ which evidently has the lowest cross-sectional diameter was observed to have highest frequency of $325 \mathrm{~Hz}$ as its first harmonic. The frequency of the other stings has been listed in Table 3. The most fundamental harmonic for a guitar string is the harmonic associated with a standing wave having only one antinode positioned between the two nodes on the end of the string. This would be the harmonic with the longest wavelength and the lowest frequency. A complete wave starts at the rest position, rises to a crest, returns to rest, drops to a trough, and finally returns to the rest position before starting its next cycle. In this condition, there is only one-half of a wave within the length of the string. This is the case for the first harmonic or fundamental frequency of a guitar string.

Table 2. FFT analysis parameters.

\begin{tabular}{cc}
\hline Parameter & Value \\
\hline Sampling Frequency & $44,100 \mathrm{~Hz}$ \\
Block Size & 1024 \\
Averaging & Instantaneous \\
Amplitude Scaling & Peak \\
\hline
\end{tabular}

Table 3. Fundamental frequency of strings.

\begin{tabular}{cc}
\hline String & Frequency $(\mathrm{Hz})$ \\
\hline E & 79 \\
A & 108 \\
D & 140 \\
G & 194 \\
B & 242 \\
e & 325 \\
\hline
\end{tabular}




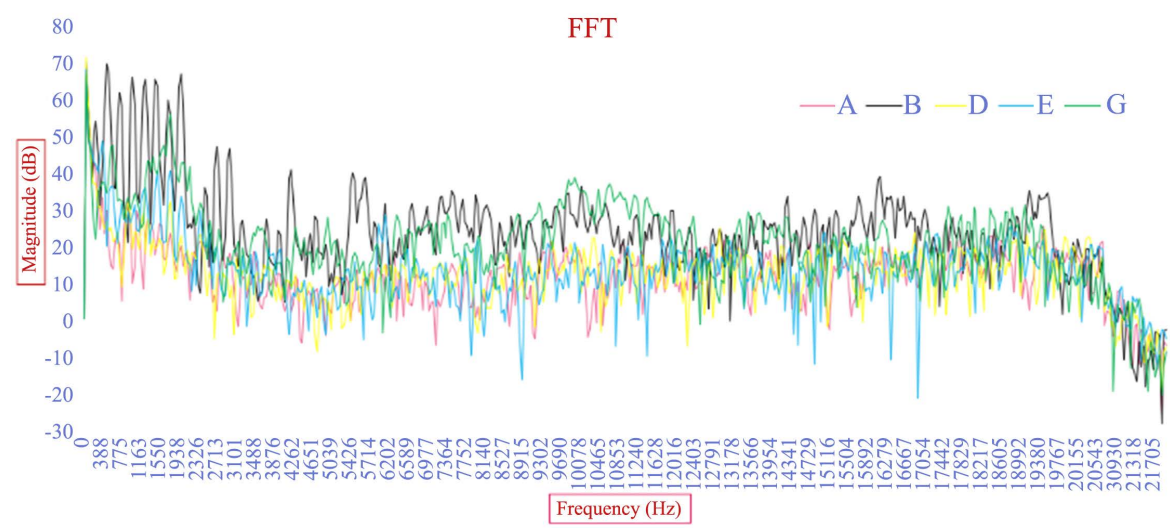

Figure 7. FFT plot of tuned strings.

The frequency of the second harmonic is two times the frequency of the first harmonic as indicated in the FFT Spectrum of string $B$ (Figure 9). Subsequently, when frequency is closely observed, they follow a trend where the frequency of the $\mathrm{n}^{\text {th }}$ harmonic (where " $\mathrm{n}$ " represents the harmonic order of any of the harmonics) is " $n$ " times the frequency of the first harmonic.

$N^{\text {th }}$ Harmonic Frequency $=($ Fundamental Frequency $){ }^{*} N^{\text {th }}$ harmonic (6)

It also means that the thin string will sound one octave above the thicker one. This was noted and recorded in the $1 / 3^{\text {th }}$ octave plot seen in Figure 8 . The spectrum distribution of string " $B$ " is taken separately and represented for a better understanding (Figure 9). Its first harmonic or Fundamental frequency was observed at $242 \mathrm{~Hz}$, second harmonic at $484 \mathrm{~Hz}$, third at $726 \mathrm{~Hz}$ and so on. This follows the trend mentioned in Equation (6).

For further study the modification of the frequencies was done to analyse inharmonicity. Each partial of the given recording was analysed in the zoomed frequency domain, tuning the fundamental frequency and the inharmonicity factor so that the main spectral peak of the partial remains in the middle of the zoomed band. Then mapping the partial goes back to the original frequency domain by moving the modal frequency to a new desired frequency. This was repeated for each partial and the resynthesized partial components were recorded.

1/3 Octave Band (Tuned)

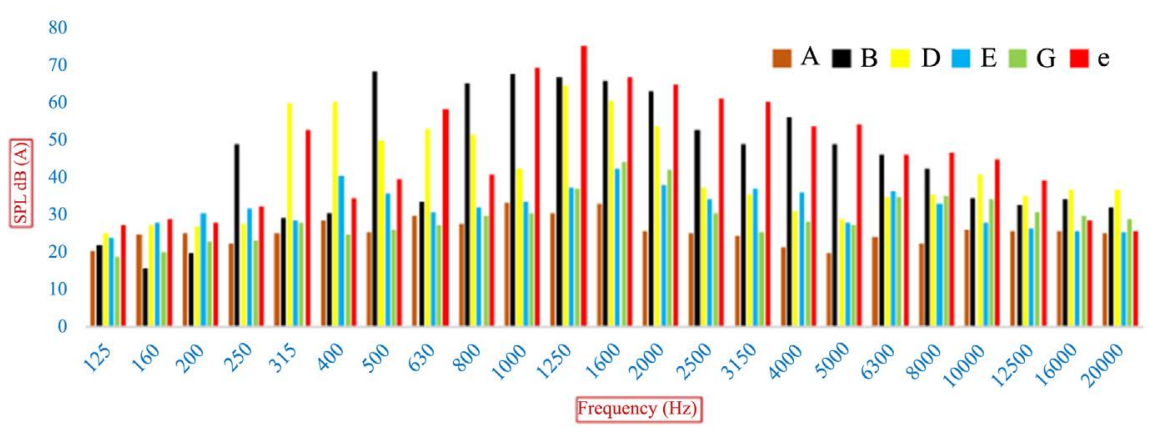

Figure 8. 1/3 octave bands of tuned strings. 


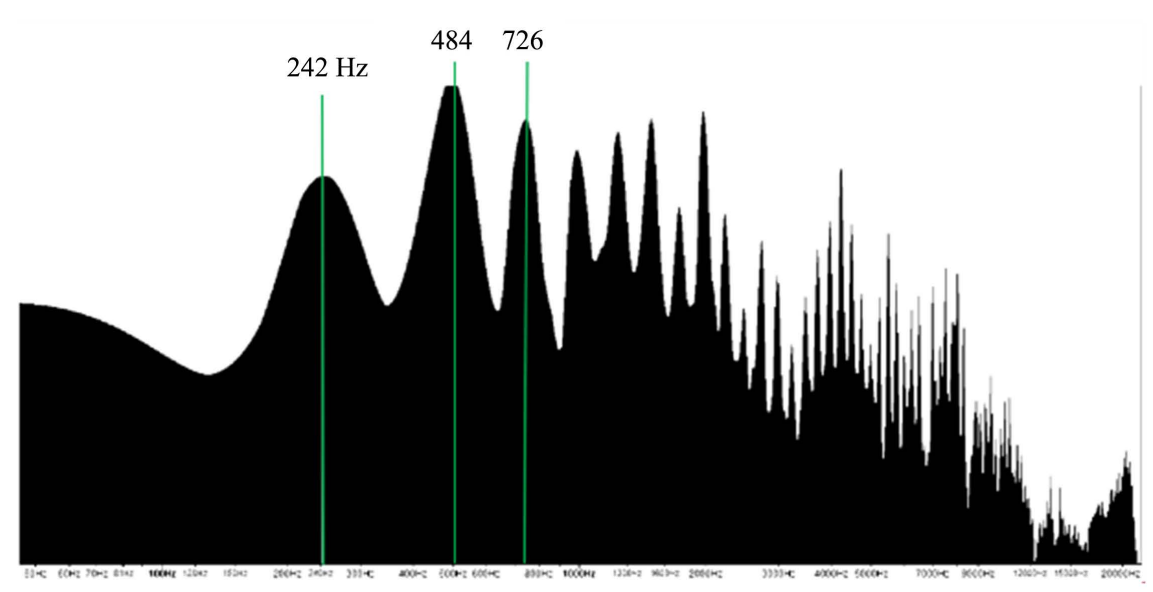

Figure 9. FFT spectrum of tuned "B" strings.

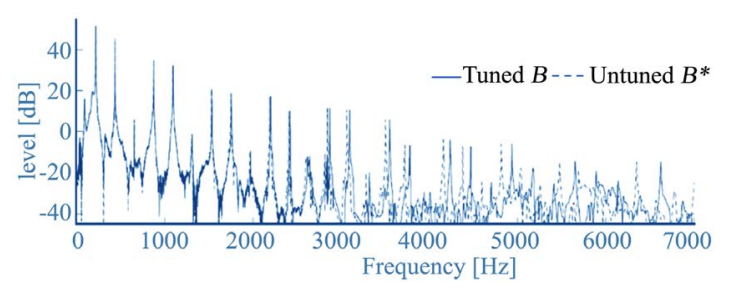

Figure 10. FFT spectrum of untuned (in-harmonic) "B” strings.

Resynthesizing the partials also at the original frequencies and subtracting this from the original signal resulted in a residual signal. Resynthesizing the new modified version from the frequency shifted partials and the residual for a new inharmonicity. Figure 10 depicts an example of plucked string spectra of " $B$ " string for the original in-harmonic recorded sound and the resynthesized harmonic version of it.

\section{Conclusion}

The finite-element and the modal analysis methods have been applied to the resonance box of a guitar with the purpose of analysing its vibrational behaviour. In this way, mode evolution can be tracked, establishing the influence of each component on the final box. Also, Fourier spectra approaches to time and frequency analysis is done. Fourier spectra identify spectral peaks within entire musical signals, while spectrograms and scalograms provide two different ways of capturing the time-frequency content of a musical signal. Some basic applications to music were also discussed. Further, the perceptibility of inharmonicity in the acoustic guitar was studied in string " $B$ " guitar tones whose inharmonicity could be accurately controlled. However, perception seemed to be strongest at the very beginning of the sound and was interfered by the presence of the plucking transient. This is understandable because the most inharmonic higher partials decay fast in string instrument tones and become easily masked by the plucking transient. Further research could be carried on the different approaches to frequency analysis involving spectrograms and scalograms. 


\section{Conflicts of Interest}

The authors declare no conflicts of interest regarding the publication of this paper.

\section{References}

[1] Romá, M., González, L., Briones, F. (2008) Software Based Acoustic Guitar Simulation by Means of Its Impulse Response. 10th Meeting on Audio Engineering of the AES, Lisbon, 12-13 December 2008.

[2] Stanciu, M.D., Vlase, S. and Marin, M. (2019) Vibration Analysis of a Guitar considered as a Symmetrical Mechanical System. Symmetry, 11, 727. https://doi.org/10.3390/sym11060727

[3] Nicholas, N.C., Gruber, C. and Hartman, N. (2017) Optimization of Acoustic Soundboard through Modal Analysis and Material Selection, Honors Research Projects, 501.

[4] Lai, J.C.S. and Burgess, M.A. (1990) Radiation Efficiency of Acoustic Guitars. The Journal of the Acoustical Society of America, 88, 1222-1227. https://doi.org/10.1121/1.399699

[5] Russell, D. (1998) Modal Analysis of an Acoustic Folk Guitar. Ph.D. Thesis, Applied Physics, Kettering University, Flint.

[6] Russel, D. and Pedersen, P. (1999) Modal Analyses of an Electric Guitar. Kettering University.

[7] Mihălcică, M., Stanciu, M.D. and Vlase, S. (2020) Frequency Response Evaluation of Guitar Bodies with Different Bracing Systems. Symmetry, 12, 795.

[8] Stanciu, M.D., Curtu, I., Itu, C. and Grimberg, R. (2008) Dynamical Analysis with Finite Element Method of the Acoustic Plates as Constituents of the Guitar. ProLigno, 4, 41-52.

[9] Atre, M.P. and Apte, D.S. (2017) Mathematical Analysis of Acoustic Guitar Notes. International Journal of Signal Processing, 2, 21-27.

[10] Alm, J.F. and Walkerm, J.S. (2002) Time-Frequency Analysis of Musical Instruments. SIAM Review, 44, 457-476. https://doi.org/10.1137/S00361445003822

[11] Li, T. and Tang, Y. (2010) Frequency Estimation Based on Modulation FFT and MUSIC Algorithm. 2010 First International Conference on Pervasive Computing, Signal Processing and Applications, Harbin, 17-19 September 2010, 525-528. https://doi.org/10.1109/PCSPA.2010.132

[12] Lucas, C.R. and de Leon, F. (2017) Effects of Changing Material Properties on Vibration Modes of Guitar Body. IEEE International Conference on Control System, Computing and Engineering, Penang, 24-26 November 2017, 139-143. https://doi.org/10.1109/ICCSCE.2017.8284394

[13] Mirianon, F., Fortino, S. and Toratti, T, (2008) A Method to Model Wood by Using ABAQUS Finite Element Software. VTT Technical Research Centre of Finland Ltd., Helsinki.

[14] Vernet, D. (2001) Influence of the Guitar Bracing Using Finite Element Method. Technical Report, University of New South Wales and Ecole Normal Superiore.

[15] Curtu, I., Stanciu, M.D. and Grimberg, R. (2008) Correlations between the Plate's Vibrations from Guitar's Structure and Physical, Mechanical and Elastically Characteristics of the Composite Materials. AMTA 08: Proceedings of the 9th WSEAS International Conference on Acoustics \& Music: Theory \& Applications, Bucharest, June 2008, 55-60. 